\title{
The analysis of violations of the dynamic and temporary registration of utterances in children with minimal dysarthric disorders
}

\author{
Ludmila Lopatina ${ }^{1}$, Ludmila Baryaeva $^{2}$, Mariya Ivleva ${ }^{1, *}$ \\ ${ }^{1}$ Herzen State Pedagogical University of Russia, 48, Moika Emb., 191186, Saint-Petersburg, Russia \\ ${ }^{2}$ Moscow City University, 4, k. 1, 2-oi Sel'skohozjajstvennyj proezd, 129226, Moscow, Russia
}

\begin{abstract}
The article is devoted to the analysis of violations of the dynamic and temporary registration of statements for preschoolers with minimal dysarthric disorders. The results were received in the process experimentalphonetic research, involving identifying the features of these components of intonation by comparing graphs of the relative duration and intensity of sounds in children with normal speech development and with various forms of minimal dysarthric disorders. There are described the results of the experiment which is characterized the manifestations and mechanisms of violations in dynamic and temporary registration of statements in children with this type of speech dysontogenesis. There is analyzed the qualitatively heterogeneous and the variable nature of violations in the usage of phonetic level units of the language sound system (duration and intensity) by children with minimal dysarthric disorders. There is characterized the connection between the violations of dynamic and temporary registration and communicative type of statement.
\end{abstract}

\section{Introduction}

The minimal dysarthric disorders are mild severity of dysarthria, which are widespread in the population of children, but not enough studied. The deviations in the formation of the speech phonetic component, which is important for education sound form of significant units, are the leading disorders in the structure of the speech defect in children with mild dysarthria. The violations of language phonetic means in children and their secondary consequences in this form of speech pathology makes it difficult to master children of various components of language ability (lexical, morphological, semantic). In preschoolers, the language ability is formed directly in the course of verbal communication. This fact makes it necessary to take into account the dominant role of the totality of various intonation means for implementation of the speech communicative function, overcoming communicative barriers [1].

The understanding of intonation means as a complex means that are components of the plan for expressing intonation is traditional for the majority of domestic linguistic research $[2,3,4]$. The intonation means include tempo, melody, pause, duration and intensity, prosodic timbre.

\footnotetext{
* Corresponding author: m.g.ivleva@yandex.ru
} 
The units of utterance that carry a new and important information are drawn up in a slower, more thorough pronunciation as opposed to units that broadcast well familiar and less meaningful information. This is expressed in a decrease in the rate of pronunciation or in increasing of its duration. In addition, a decrease in the rate of the end of the utterance signals its completion. Duration and tempo as a means of intonation characterize relative speed of pronunciation of individual words in a syntagma or one syntagma in relation to another [5]. The semantics of the tempo is determined by the level of significance of the statement content and displaying the speed of individual events and actions. The transmission of emotions is a secondary role of tempo as an intonational means.

The content of the utterance and the function of the words are reflected on the pronunciation rate, this is manifested in following. Auxiliary words are pronounced at a faster tempo than significant, among the most significant those words are pronounced more slower which carry the main semantic load [6]. The dynamics of intensity as an energy component of intonation largely correlates with temporal means: significant and new information pronounced with greater intensity; less important and already familiar - with smaller. The intensity (or sound power) is characterized as density flow of sound energy. There are analyzed the levels, directions, the nature of the change in intensity in the theoretical sources. In accordance with this its various forms are distinguished. There are distinguished six forms of intensity taking into account the direction of change intensity: 1) constant level; 2) increase; 3) increase followed by decrease; 4) decrease; 5) decrease with subsequent increase; 6) "tremolo" (multiple decrease and increase) [7]. In terms of the nature of the change intensity, there are three forms of it: 1) expulsive (associated with short-term, energetic stress of abdominal muscles, creating a ragged, short exhalation); 2) effusive (characterized by uniform tension of the abdominal muscles, provides a calm, uniform, gradual exhalation); 3) explosive (excessive tension of the abdominal muscles leads to brief pulsations of exhalation) [8].

There is presented a similar approach in determining the forms of intensity in the research of M.V. Davydov [9]. By the nature of the change intensity, the author highlights an equal, smooth, sharp, and depending on the level of its change - high, medium and low. Within the framework of these forms, T.M. Nikolaeva [10] considers it necessary to take into account the direction of movement intensities: upward and downward.

The semantic function is the leading function for the intonation intensity. It is associated with a change in the intensity of a segment that expresses the part of the content that should be highlighted by the speaker in accordance with the communicative task [10,11].

Based on the above, we can conclude that the intensity and duration as intonation means provide in greater degree the intonation division, the transfer of the connection type of division units, in the lesser degree - the registration of the intonation type of the statement.

Currently there is a number of research describing the features of intonational components in children with minimal dysarthric disorders. The existing research are mainly characterized the features of perception and reproduction of melodic (the frequency of main tone) of the leading communicative types of statements. However, these features have not been studied in relation to other intonational components; there is not taken into account the localization of microorganic damage and the clinical form of minimal dysarthric disorder when analyzing mechanisms of their violations. Besides this, in existing research, the original data were obtained by subjective method, which consisted in the analysis of the speech signal when auditory perception. At the same time, the implementation of an objective instrumental experimental-phonetic method, involving the usage of physical measurements, is progressive in the research and correction of the disorders of the intonation components in children of this category, so this method permits to reveal their general and specific violations, their mechanism of occurrence. 


\section{Methods and materials}

The research of intonation components - duration and intensity - was carried out by the experimental-phonetic method (oscillography) in the process of children reproduction of various sentences. The research data was obtained on a probabilistic sample of two groups of children. The first group (experimental) consisted of 315 preschoolers 5.5-6.5 years old with minimal dysarthric disorders (mild dysarthria). The second group (group for comparative analysis) is included 353 children with normal speech development of the same age.

The recording of children's statements was carried out in a speech therapy room using a Panasonic L30 recorder in conditions of natural speech interference. Further the received records have been subjected to auditory and instrumental analysis. There was realized a selection of statements of various communicative focus in the process of the experimentalphonetic research: narrative, interrogative (general and special question), exclamation points, imperative. They were processed in computer system EDS. There were constructed the graphs of changes in indicators of the relative duration and relative intensity of sounds throughout speech fragment using Excel. In this article, we will consider the features of the dynamic (intensity) and temporary (duration) formulation of the statements of various communicative types. The obtained results were analyzed based on comparison of the obtained graphs of the studied indicators.

\section{Results}

The research of these indicators allows us to state that children with normal speech development show characteristic features in the formulation of completed narrative utterances, which are inherent for children's speech. In particular, the beginning of the syntagma was characterized by more intensity than its end; the main-stressed vowel was more highlighted in intensity compared to less powerful pre-stressed and post-stressed vowels. There are the features of the dynamic and temporal components of intonation means of formalizing statements in children with minimal dysarthric disorders. These features are caused by the pathological state of the muscles of the speech apparatus. The following was revealed while analyzing and comparing graphs of completed narratives statements in children with minimal dysarthric disorders. The children of the experimental group had a characteristic feature of the violation in reproduction of the studied intonation means. This feature is manifested in the fact that the distribution of intensity and duration values often do not correlate with verbal or syntagmatic stress. It is implemented in an approximately the same accentuation of both stressed and unstressed syllables. The mistakes occur when reproducing both phrasal and verbal stress due to the fact that the highlighting of the mainstressed vowel differs insignificantly from unstressed parts of the statement. This, in turn, makes difficult the perception of the child's speech by people around him, negatively affects the ability of accurately transmitting the statement meaning.

The research has shown that the maximum values of intensity rarely appear at the beginning of syntagmas in preschoolers with minimal extrapyramidal dysarthria. In some statements, there was no decrease in intensity and duration by the end of the phrase, or its degree was insignificant. The limited intensity range also affects negatively on the quality of intonational formulation of statements and significantly differs from that in the speech of children with normal speech development. The highest intensity indicators were recorded on the stressed vowel, but not on the main-stressed vowel, which indicates a violation in the usage of phrasal and verbal stress in the speech of children. The indicators of the duration for stressed vowels are even less significant than the intensity indicators. 
The duration does not accept significant participation in the accentuation of the stressed parts of the statement.

Children with minimal pseudobulbar dysarthria are characterized by using a limited range of intensity when implementing complete narrative types of utterances. There are examples of a sudden increase of intensity indicators on the post-stressed vowel, which are not related to highlighting the main-stressed vowel sound.

Such cases may be associated with an involuntary increase of tension of the vocal folds, which is typical for pseudobulbar dysarthria. Such phenomena lead to mistakes in the reproduction of phrasal and verbal stress, and also to the rapid depletion of the voice. In this case, after the maximum value of the intensity, there is a sudden decrease in its indicators by the end of phrases. Throughout the whole sentence the indicators of the relative duration of stressed vowels slightly differ from those for unstressed vowels. That is the duration does not adequately provide the opposition of stressed and unstressed syllables.

The usage of a larger range of intensity is typical for implementing the completed children's narratives with mixed forms of minimal dysarthric disorders, but in a number of cases there is an increased emphasis of not only stressed, but also unstressed parts of phrases by means of intensity and duration. Such defects in the formulation of the statement lead to the appearance of scansion and tension in speech.

The dynamic and temporary formulation of narrative statements in children with minimal cortical dysarthria is generally closer to such in speech in norm. There is a large intensity at the beginning of the syntagma than in its ending. Children use a wider range of intensity to create dynamic changes within the utterance. However, the distribution indicators of duration and intensity depends not always on the position of the verbal stress, which often leads to its violation.

The dynamic and temporary formulation of interrogative statements in preschoolers with minimal dysarthric disorders has specific characteristics in compare with children with normal speech development. In children with normal speech development, an increase in values of duration and intensity falls on the stressed vowel. It especially clearly marked on the vowel that is under syntagmatic stress, which is emphasized with a greater force and the duration of the utterance. The intensity values at the beginning of phrases exceed those at the end, that generally corresponds to the trend in the adult's speech. The level of decreasing the intensity towards the end of the phrase in general questions is significant in the same way as in the reference models of interrogative statements of adults.

Similar phenomena is observed with formulation of special questions. In children with normal speech development, the beginning of utterances is characterized by more high intensity values, which are noticeably decreasing towards the end. The highlighting of the main-stressed vowel is carried out using an increase of duration and intensity of pronunciation; a stressed vowel of the highlighted by the meaning word also has the higher values of intensity.

The possibilities of the dynamic and temporary formulation of interrogative statements by children with various forms of minimal dysarthric disorders are qualitatively heterogeneous.

There is a tendency to increased highlighting as stressed and unstressed parts of statements in children with minimal extrapyramidal dysarthria, when implementing the general questions, as well as in completed narrative statements. Thus, there are specific signs of scansion; both stressed and unstressed vowels are highlighted by both duration and intensity. At the same time, the insignificance of changes in the studied characteristics during the whole utterance is clearly noticeable. By the end of the phrase, there is an increase of intensity, which indicates an increase of tension. The similar picture (scansion and tension when pronouncing both stressed and unstressed parts of the phrase) was also observed in formulatinig the special questions. 
The dynamic and temporary formulation of the question phrases in children with minimal pseudobulbar dysarthria is characterized by insignificant increase in intensity on the mainstressed vowel, although in general it is close to that in speech of preschoolers with normal speech development. The unstressed vowel sounds often correspond to large values of the intensity and duration than stressed vowel sounds. Such phenomena lead to "smoothness" of the dynamic contours of common questions in children with this form of minimal dysarthric disorder in the comparison with children without speech pathology. When reproducing special questions, these children rarely use models whose formulation correspond the same models in the speech of children with normal speech development and adults. In such cases, the highest values of intensity and duration do not occur at the beginning of syntagmas. By the end of the phrases the relative duration of sounds increases; the intensity decreases. While in children with normal speech development, the values of these indicators change accordingly each other.

When implementing general questions, preschoolers with mixed forms of dysarthric disorders use a smaller intensity range, than children with normal speech development. Moreover, in the formulation of general questions they have a tendency to increase the duration and intensity on stressed vowel sounds; there is a decrease in intensity towards the end of utterances, which is also typical for the implementation of statements in children without speech pathology. The specifics of the formulation of general questions by children with mixed forms of minimal dysarthric disorders consists in the presence of frequent cases of highlighting with using the intensity and duration on each word in the phrase. This kind of statements implementation can lead to difficulties in the reproduction of syntagmatic stress, as well as create the effect of speech tension.

In some cases, when children with minimal cortical dysarthria formulate the general questions, there is a violation of stress when the maximum value of intensity and duration falls on the first post-stressed vowel, not the main one, which is under the syntagmatic stress. These children use more wide range of the intensity in comparison with that of children with normal speech development. In some cases, when implementing special questions, the indicators of intensity and duration are close to each other both at the beginning and at end of the phrase. Some phrases show a tendency to accordance of increasing the duration and intensity and the main-stressed vowel sound in the syntagma, which is typical for children without speech pathology.

Children with normal speech development during the implementation of the exclamation statements are characterized by the usage of a wide range of intensity. The greatest duration of the main-stressed vowel sound in syntagma is characterized for the formulation of the exclamation phrases. The highest values of intensity and duration are marked on a stressed vowel.

The formulation of the exclamation statements by children with minimal dysarthric disorders are characterized by specific features. All the exclamation phrases, which were selected for analysis and obtained by repeating children after experimenter, were not recognized by ear as exclamation.

The comparison of the graphs of the relative intensity and duration of sounds demonstrated that the dynamic and temporary formulation of these phrases in generally more consistent with the type of complete narrative.

In the statements of children with minimal extrapyramidal dysarthria maximum intensity and duration values are noted at the beginning of the statements on the main-stressed vowel of the only intonational center; there is the decrease of the intensity towards the end, which is not typical for this intonation construction. The changes in the duration of vowel are insignificant; the signs of scansion are noted. There is the maximum of the intensity value at the beginning of the syntagma, on the main vowel in the statements of preschoolers with minimal pseudobulbar dysarthria. The significant increase in intensity fixed not only on 
stressed, but also on unstressed vowels, which leads to tension in speech. The decrease of the intensity appears towards the end of the phrase. The relative duration of sounds increases to the end of the phrase, as with the implementation of the interrogative statements. This causes a slowdown in the tempo of speech.

There was noted the change in the studied characteristics in the statements of children with mixed forms of minimal dysarthric disorders, which is more characteristic of a complete narrative than of exclamation type of phrases. In these cases, both intensity and duration are involved in highlighting the main-stressed vowel. It is noted the decrease of intensity by the end of the phrase. The post-stressed vowels of the intonation center are characterized by the less strength and duration, than stressed vowels, that is also typical of the completed type of utterance.

The phrases in children with minimal cortical dysarthria were also not identified by ear as exclamation one. The analysis of the dynamic and temporal changes in the implementation of the statement demonstrated that it is more corresponds to the type of complete utterances. Namely, the main-stressed vowel in the only intonation center is highlighted by both the intensity and duration of pronunciation; there is a significant reduction of these characteristics on the post-stressed part.

Children with normal speech development use models of the dynamic and temporary formulation of imperative utterances that are also found in the speech of adults. The beginning of the syntagma is characterized by the greater intensity than its ending.

The essential increase in the relative duration of a vowel under syntagmatic stress is noticed, this is typical for incomplete statements as opposed to complete narratives. As in standard speech models of adults, in children without speech pathology the maximum intensity value is marked on the main-stressed vowel, and the unstressed vowels of word have high values of intensity.

There was noticed the significant violations in the usage of the studied components of intonation in children with minimal extrapyramidal dysarthria, when implementing the spontaneous motivating statements. The beginning of the syntagmas is characterized by low rates of the intensity and duration, at the same time a low level of the intensity remains when pronouncing the whole phrase. The sudden changes in the duration values indicates the increasing tension and slowness of the speech. The noticeable scansion of the pronunciation is illustrated by the distribution of the intensity and duration values, which is not associated with the phrasal or verbal stress.

A larger range of the intensity is characteristic for the implementation of imperative statements by preschoolers with minimal pseudobulbar dysarthria. At the same time, they have violations of the verbal stress, which appears because of increased highlighting the unstressed syllables of words by both the duration and intensity.

The implementation of the imperative statements in children with mixed forms of minimal dysarthric disorders is close to the norm models; this is expressed in the usage of a larger range of intensity. For example, the higher intensity and duration values are characterized for appeal as part of a two-syntagma utterances; there is noticed an increase in the values of these characteristics and on the main-stressed vowel of the second syntagma. Along with this, in such children there are cases of the violation of the verbal stress, which are manifested an increase in the intensity of unstressed syllables.

\section{Discussion}

The data, which were obtained experimentally in the process of experimental phonetic research (oscillography), indicate that there are the significant differences in the dynamic and temporal formulation of various communicative types of statements by children with minimal dysarthric disorders and their peers without speech pathology. The limited possibilities in 
using and timely scansion such components of intonation as intensity and duration make it difficult to accurately transmission the characteristic signs of various intonation structures, a clear opposing the stressed and unstressed parts of the phrase, which influence negatively on the perception and assessment of their communicative types by the surrounding people. There are discovered both general and specific features of the dynamic and temporary formulation of various communicative types of statements by preschoolers with various forms of minimal dysarthric disorders in the researh. The qualitative heterogeneity in the studied indicators is due to the localization of brain damage.

\section{Conclusion}

There are revealed the features of the studied intonation means in preschoolers with minimal dysarthric disorders, when making statements in comparison with those in the speech of children without speech pathology. The models of various communicative types used by children with speech pathology are not correspond to the patterns typical for children with normal speech development and adults by the distribution of intensity and duration values. They are characterized by the usage of the limited range of the intensity. The distribution of the intensity values and duration is often not associated with the verbal and syntagmatic stress.

Children with minimal dysarthric disorders have various violations of the dynamic and temporary formulation of the statements, which have various degree of the severity. The most significant violations of the intensity and duration were recorded in children with minimal extrapyramidal and pseudobulbar dysarthria. An intermediate position is occupied by children with mixed forms of minimal dysarthric disorders. The dynamic and temporary formulation of statements by children with minimal cortical dysarthria is closest to occurring patterns in the speech of children without speech disorders.

Preschoolers with minimal dysarthric disorders are characterized by qualitatively heterogeneous manifestations of violations. The emphasis on the stressed parts of the phrase is disturbed more often in children with minimal extrapyramidal and pseudobulbar dysarthria; there are noticed scansion, tension and slowing down the tempo of speech towards the end of the statements of all studied communicative types. The dynamic and temporary formulation of completed narratives by preschoolers with minimal cortical dysarthria is inherently close to the norm. The preschoolers use a wide range of the intensity to provide the dynamic changes within a phrase, when implementing other intonational types of utterances. However, this wide range is not always sufficient to transmit an accent contour of interrogative and exclamation phrases. In children with mixed forms of minimal dysarthric disorders there are features inherent in all forms, in various combinations.

\section{References}

1. L.V. Lopatina, Speech therapy work with preschool children with mild dysarthria (Dobryi mir, Moscow, 2015)

2. L.V. Bondarko, The phonetics of modern Russian language (SPbGU, Saint-Petersburg, 1998)

3. L.V. Bondarko, L.A. Verbitskaya, N.V. Gordina, The basics of general phonetics (SaintPetersburg, SPbGU, Moscow, Academia, 2004)

4. The linguistic encyclopedic dictionary (Soviet encyclopedia, Moscow, 1990)

5. L.R. Zinder, General phonetics and selected articles (Academia, Saint-Petersburg, SPbGU, Moscow, 2007) 
6. L.K. Tseplitis, The analysis of speech intonation (Znanie, Riga, 1974)

7. S.M. Volkonskii, Expressive word. The research and leadership experience in the field of mechanics, psychology, philosophy and esthetics of speech in life and on scene (Lenand, Moscow, 2019)

8. H.P. Constans, Studies in experimental phonetics 27, 8-17 (1966)

9. M.V. Davydov, The paralinguistic functions of super-segmental means of English in comparison with Russian (MGU imeni M.V. Lomonosova, Moscow, 1965)

10. T.M. Nikolaeva, The semantics of the accentuation (Librocom, Moscow, 2010)

11. N.D. Svetozarova, Intonation in a literary text (SPbGU, Saint-Petersburg, 2000) 